\title{
A Multiparameter Model for the Correlation Between CT Hounsfield Unit and Blood Components
}

\author{
Pop Marian ${ }^{1}$, Pop Raluca2*, Bacarea Anca ${ }^{3}$ \\ ${ }^{1}$ PhD Student, University of Medicine and Pharmacy Tirgu Mures, Romania \\ 2 Department of Research Methodology, University of Medicine and Pharmacy, Tirgu-Mures, Romania \\ 3 Department of Pathophysiology, University of Medicine and Pharmacy, Tirgu-Mures, Romania
}

\begin{abstract}
Objective: To search for a dependency between blood Hounsfield Units values as measured by Computed Tomography and blood components with regards to hemoglobin oxygenated status, demographic data and acquisition parameters. Methods: A retrospective study included a convenience sample of 144 SCJU patients who underwent routine thoracic CT examinations (Siemens Somatom AS 64 or 128 MDCT) in 2014 and had laboratory examinations done in nearby time. Variables included age, sex, mA, mV, aortic HU values, PA HU values, RBC, HTC, HGB, PLT, WBC blood sugar, PT and INR. Statistical analysis was performed using R version 3.1.1 and MedCalc 12.5 with a statistical significance factor of 0.05. Results: The examined patients had an average age of 56.25, with a predominance of males (M:F=1.62). While there was a minor difference between $\mathrm{HU}$ values in Males vs Females (1.01 HU) it was proven as statistically significant ( $p=0.0051)$. No statistical significant difference between oxygenated/non-oxygenated blood $(p=0.9636)$. Regression analysis found positive correlation between HU values and RGB, HGB and HCT with a significance level below 0.0001, the strongest being for the HGB level. Conclusions: While $\mathrm{HU}$ values seem to carry multifactorial sources and the red blood cells being the most important an importance should be given to the fibrinoid plasma components which appear to bear negative impact.
\end{abstract}

Keywords: computed tomography, blood, Hounsfield unit

Received: 09 February 2016 / Accepted: 21 March 2016

\section{Introduction}

Computed tomography (CT) uses a computer to reconstruct a cross-sectional image of the body from measurements of $x$-ray transmission through thin slice of patient tissue [1]. One or more detectors [2] are used for detection of the $\mathrm{x}$-ray beam attenuated by absorption and scatter as it passes through the patient. CT numbers are assigned to each pixel in the image by a computer algorithm that uses as data these measurements of transmitted $\mathrm{x}$-rays $[1,3]$. CT pixel numbers are proportional to the difference in average $\mathrm{x}$-ray attenuation of the tissue within the voxel and that of water. A Hounsfield unit $(\mathrm{H})$ scale, named for Sir Godfrey $\mathrm{N}$. Hounsfield, the inventor of CT, is used. Water is assigned a value of $0 \mathrm{H}$, with the scale extending from 1,024 $\mathrm{H}$ for air [1]. The excellent contrast resolution of current CT scanners can detect differences in contrast of less than $5 \mathrm{H}(<0.5 \%)[4]$.

Different body structures $\mathrm{HU}$ values can be calculated theoretically using available data [5]. Multiple studies[4,6] have tried to asses anemic status using only HU data on non-enhanced CT exams but none tried to evaluate the weight of different blood components.

\section{Methods \\ Patients}

In our single-center study were included a number of 144 patients who underwent routine unenhanced CT thoracic

* Correspondence to: Raluca Pop

E-mail: ralucapetri@yahoo.com examinations for various indications and had laboratory examinations being done in the nearby time.

Patients with active bleeding, shock or diagnosed with pulmonary thromboembolic disease following contrast administration were not included in our analysis. Institutional approval was acquired for the current study.

The study used data gathered from unenhanced CT examinations done on 64 or 128 slice CT system (Siemens Somatom AS), using a standard thoracic acquisition protocol (100-140 kV and modulated $\mathrm{mA}$ ), with reconstructions performed using either a soft (B30/B31) or high resolution (B70/B80) body standard kernel. No contrast medium was given for any patient before or during the examination.

For each patient a general radiologist with more than 5 years of experience selected the slices where the required anatomical landmarks were properly represented. A number of 16 measurements were performed using a circular region of interest (ROI) with $1 \mathrm{~cm} 2$ area using 5 different slices in the following anatomical structures (cranio-caudal):

1. Aortic arch (2 measurements) (figure 1)

2. Ascending and descending aorta (2 measurements) (figure 2)

3. Ascending and descending aorta, left pulmonary artery (4 measurements) (figure 3 )

4. Ascending and descending aorta, right pulmonary artery (4 measurements) (figure 4)

5. Ascending and descending aorta, main pulmonary artery ( 4 measurements) (figure 5)

The slices were selected so that they would be artefactfree, with no beam-hardening, streak or motion artifacts. 


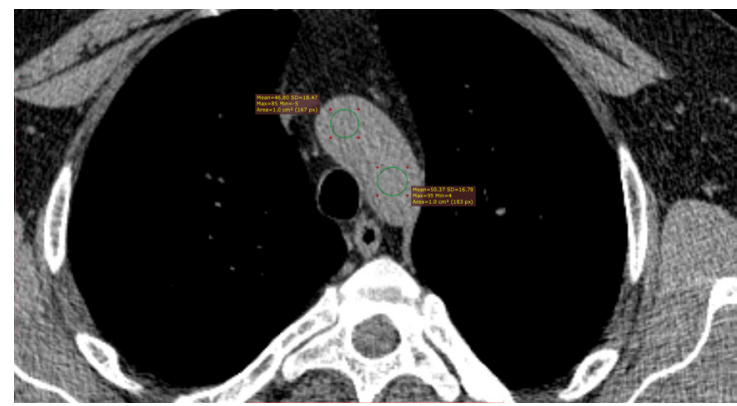

Fig. 1. Aortic arch slice

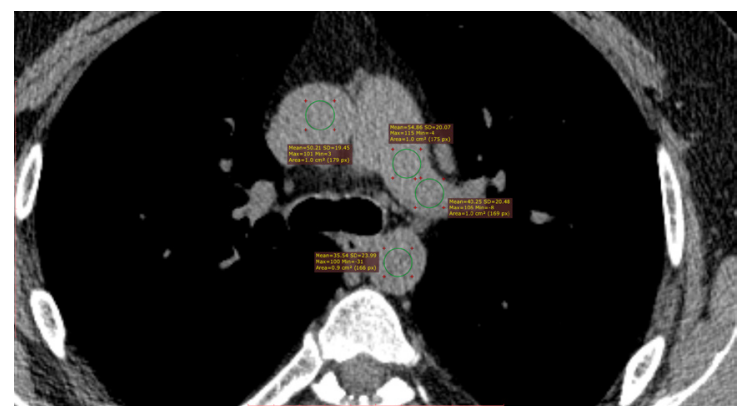

Fig. 3. Ascending, descending aorta and left pulmonary artery slice

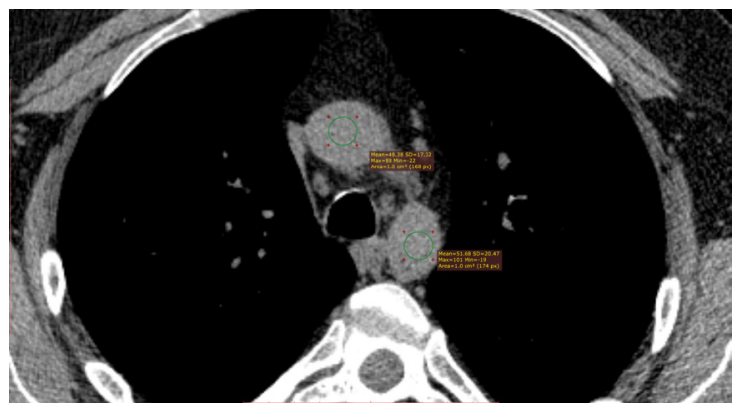

Fig. 2. Ascending and descending aorta slice

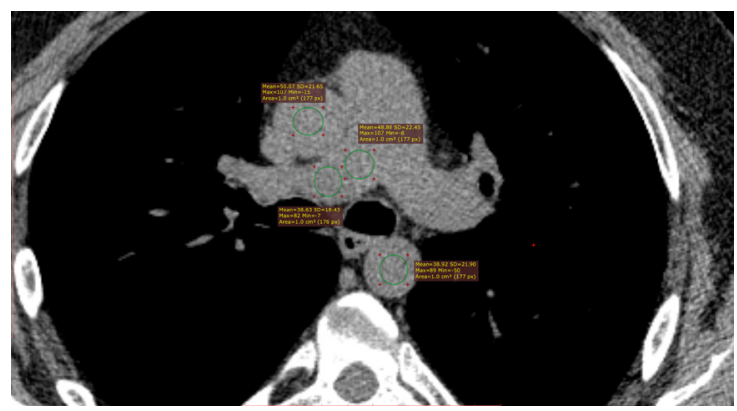

Fig. 4. Ascending, descending aorta and right pulmonary artery slice

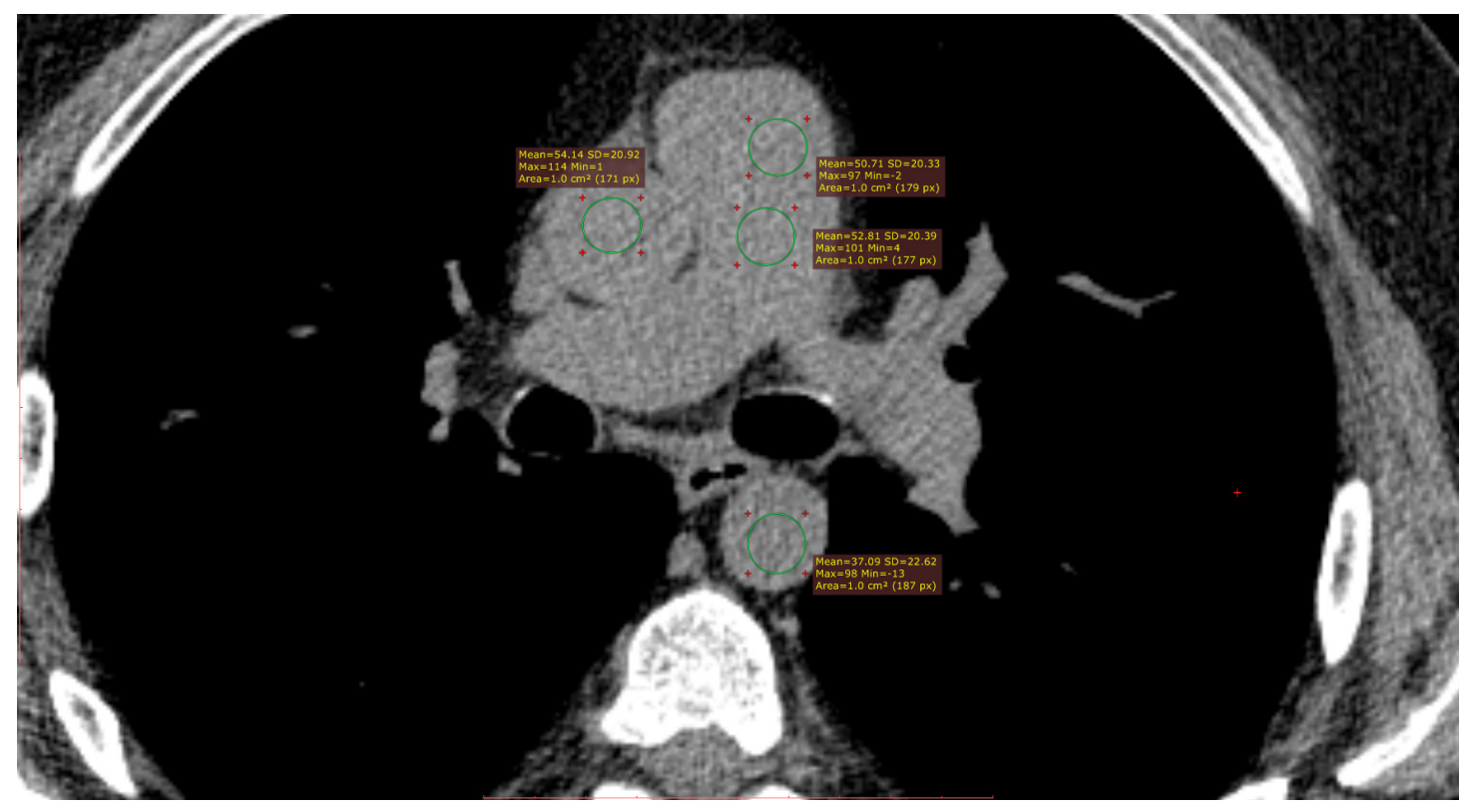

Fig. 5. Ascending, descending aorta and main pulmonary artery slice

All CT examinations were performed using soft tissue window, with the radiologist unaware of the laboratory findings.

The recorded variables in the database included also the age, sex, $\mathrm{mA}, \mathrm{mV}$, red blood cell, hematocrit, hemoglobin, platelets, white blood cell, blood, glycaemia, prothrombin ratio and international normalized ratio (INR).

\section{Statistics}

Statistical analysis was performed using $\mathrm{R}$ version 3.1.1 and MedCalc 12.5 with a statistical significance factor of 0.05 .
The values used for $\mathrm{HU}$ values were computed as average of aortic measurements, respectively of pulmonary artery measurements. Using linear regression analysis, the correlation between average blood $\mathrm{HU}$ values and each variable was evaluated.

\section{Results}

Our study included a number of 144 patients ages 7 to 88 years (average age 56.25) with a higher number of males (M:F=1.62).

We found no significant difference between the $\mathrm{HU}$ values of aortic and pulmonary blood $(\mathrm{p}=0.9636)$. 
Subgroup analysis revealed that while there was a minor difference between $\mathrm{HU}$ values in Males Vs Females (1.01 $\mathrm{HU}$ ) it was statistically significant ( $\mathrm{p}=0.0051$ ) (figure 6).

Regression analysis found positive correlation between HU values and RGB, HGB and HCT with a significance level below 0.0001, the strongest being for the HGB level. As expected, the glycaemia and white blood cells had no influence on $\mathrm{HU}$ values (table I, II, figure 7-9).

With a regression equation $\mathrm{y}=20.0350+1.7768{ }^{*} \mathrm{x}$ $(\mathrm{p}<0.001)$ for each $1 \mathrm{~g} / \mathrm{L}$ increase of the hemoglobin level the $\mathrm{HU}$ values would increase with 1.77 ; with average val-

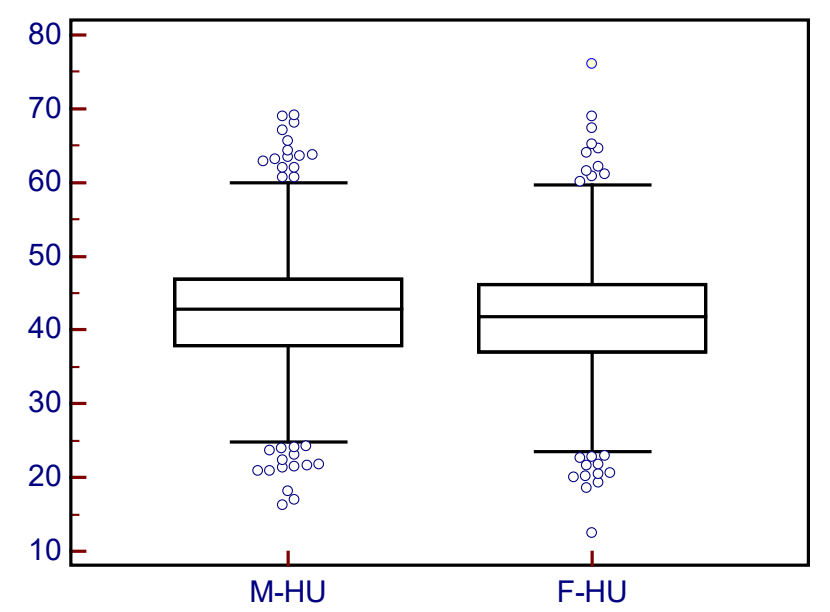

Fig. 6. Comparison between male (M) and female (F) Hounsfield Unit average values. There is a small but significant difference between them.

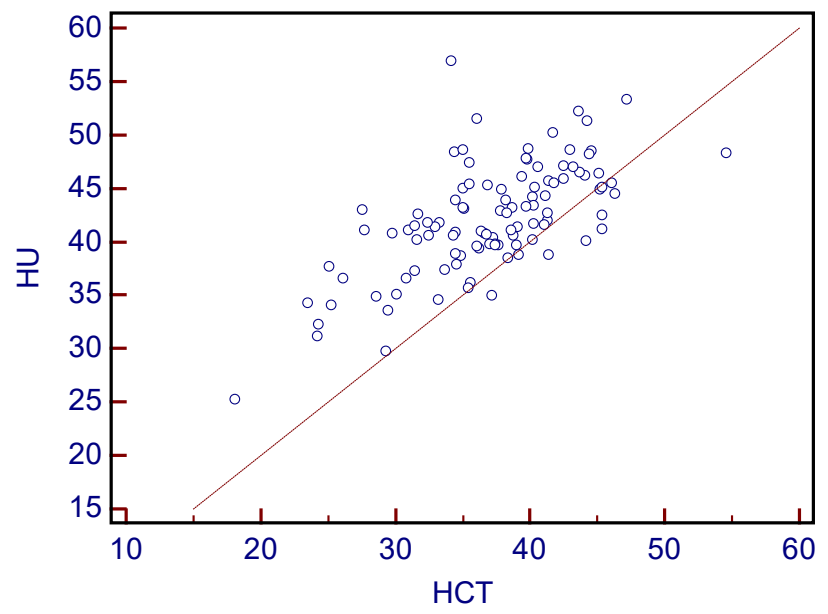

Fig. 7. Correlation between $\mathrm{HU}$ and hematocrit in all patients.

Correlation coefficient $r=0.6606$; Significance level $P<0.0001$; $95 \%$ Confidence interval for $r=0.5369-0.7565$ ues of hemoglobin 12 to $17 \mathrm{~g} / \mathrm{L}$ corresponding to a $\mathrm{HU}$ range of 41.36 to 50.24 .

Multiple regression found that both hematocrit and hemoglobin acted as positive coefficients in the equation while prothrombin time acted as a negative coefficient. The determination coefficient was in both cases a weak one (0.4383 and 0.3888) (table III, IV).

Multiple regression analysis kept the previous positive correlations and found negative correlations for INR and $\mathrm{PT}$, while the RBC and platelets had no influence. As expected, the glucose and white bodies had no influence on HU values.

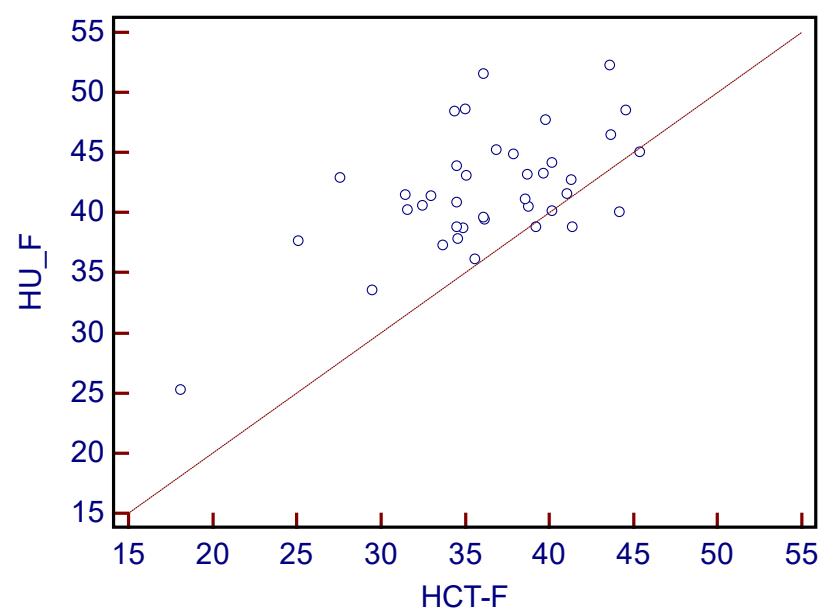

Fig. 8. Correlation between $\mathrm{HU}$ and hematocrit in women. Correlation coefficient $r=0.5880$. Significance level $P=0.0001$. $95 \%$ Confidence interval for $r=0.3345-0.7621$

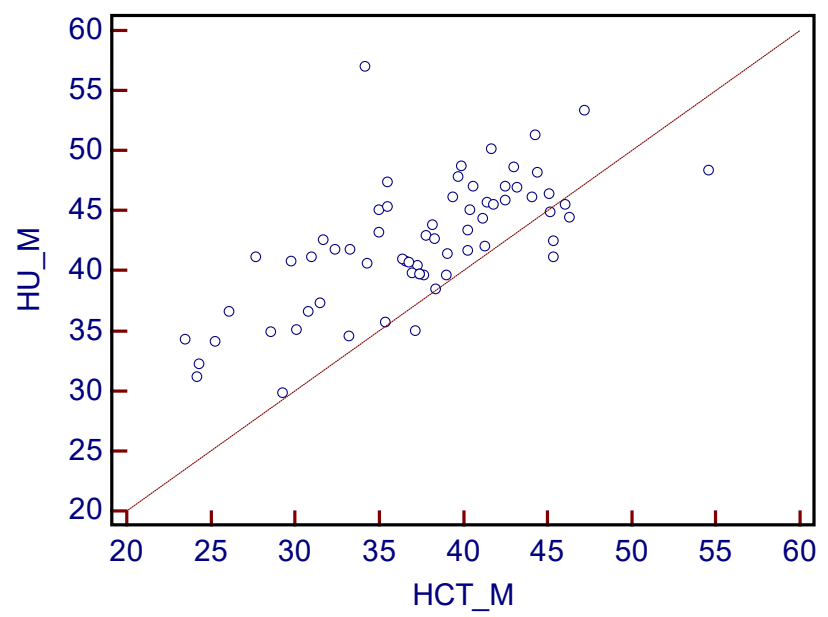

Fig. 9. Correlation between $\mathrm{HU}$ and hematocrit in men. Correlation coefficient $r=0.6943$, Significance level $\mathrm{P}<0.0001,95 \%$ Confidence interval for $r=0.5437-0.8017$

Table I. Correlation coefficient between blood components and average HU value.

\begin{tabular}{|c|c|c|c|}
\hline Variable & Correlation coefficient $(r)$ & Significance level & 95\% confidence interval \\
\hline WBC & -0.01071 & $\mathrm{P}=0.9137$ & -0.2020 to 0.1813 \\
\hline $\mathrm{RBC}$ & 0.5844 & $P<0.0001$ & 0.4423 to 0.6979 \\
\hline HGB & 0.6951 & $P<0.0001$ & 0.5808 to 0.7825 \\
\hline HCT & 0.6606 & $P<0.0001$ & 0.5369 to 0.7565 \\
\hline PLT & -0.05952 & $P=0.5464$ & -0.2484 to 0.1337 \\
\hline INR & 0.07820 & $P=0.5108$ & -0.1547 to 0.3028 \\
\hline Glycaemia & -0.03373 & $P=0.7816$ & -0.2666 to 0.2029 \\
\hline
\end{tabular}


Table II. Average hemoglobin levels for men and women grouped by Hounsfield Unit value range.

\begin{tabular}{ccccc}
\hline $\begin{array}{c}\text { Hounsfield values } \\
\text { range }\end{array}$ & $\mathrm{N}$ & $\begin{array}{c}\text { Women } \\
\text { Mean Hg level, } \\
\mathrm{g} / \mathrm{L}\end{array}$ & $\mathrm{N}$ & $\begin{array}{c}\text { Men } \\
\text { Mean Hg level, } \\
\mathrm{g} / \mathrm{L}\end{array}$ \\
\hline$<35$ & 3.00 & 7.96 & 9.00 & 9.48 \\
$35-40$ & 14.00 & 11.43 & 14.00 & 11.49 \\
$40-45$ & 25.00 & 12.31 & 37.00 & 12.78 \\
$45-50$ & 11.00 & 13.57 & 25.00 & 14.03 \\
$>50$ & 2.00 & 13.30 & 4.00 & 14.40 \\
\hline
\end{tabular}

Table III. Multiple regression coefficients when asserting HU values, HGB and PT.

HU, HGB and PT. R2 $=0.4383$

\begin{tabular}{lccc}
\hline Independent variables & Coefficient & Std. Error & $\mathrm{P}$ \\
\hline (Constant) & 22.7728 & & \\
HGB & 1.6578 & 0.2346 & $<0.0001$ \\
PT & -0.06835 & 0.03589 & 0.0611 \\
\hline
\end{tabular}

Table IV. Multiple regression coefficients when asserting HU values, HCT and PT.

\begin{tabular}{lccc}
$\mathrm{HU}, \mathrm{HCT}$ and PT. R2 $=0.3888$ & & \\
\hline Independent variables & Coefficient & Std. Error & $\mathrm{p}$ \\
\hline (Constant) & 24.4533 & & \\
$\mathrm{HCT}$ & 0.5141 & 0.08082 & $<0.0001$ \\
PT & -0.06980 & 0.03745 & 0.0667 \\
\hline
\end{tabular}

\section{Discussion}

Our research confirms the long-held credence that blood radiodensity correlates with the level of hemoconcentration.

We demonstrated that CT Hounsfield unit values are highly positive correlated with both HGB and HCT levels while PT and INR have a negative low correlation. As expected glycaemia have no correlation. The findings are interesting because they open the possibility towards assessing the anemic status based only on the blood HU values.

Since the beginning of working with CT New and Aronow [7] demonstrated an in vitro correlation between JU and HCT.

Similar correlation has been noted also in another study where HGB and HCT were found to have linear correlation to $\mathrm{HU}$ values by using a smaller sample than ours (22 patients who underwent unenhanced CT of the chest) [8].

Using data collected from aorta was fast and feasible but other locations are also used. In a study by Black et al. [9] it was found a direct correlation between the cerebral venous sinus density on noncontrast CT and hematocrit.

Also on a study based on evaluating the venous blood in the cranial venous drainage[10] it was found that although a correlation between anemia and HU levels is not strong enough to offer a definite diagnosis a larger sample size is likely to strengthen the predictive value of an $\mathrm{HU}$ cutoff value.

There are several limitations of this study. The relative small number of cases included and the fact that all the samples were obtained from acquisitions performed on a CT scanner of only one manufacturer can ask for an extension of the study. We believe that by using a retrospective study with almost 4000 samples we achieved a realistically and clinically appropriate range of patients; however, a larger data sample could be benefic for a better understanding of all the involved factors.

\section{Conclusion}

While HU values seem to carry multifactorial sources and the red blood cells being the most important an importance should be given to the fibrinoid plasma components which appear to bear negative impact.

\section{Acknowledgement}

Partly financed by University of Medicine and Pharmacy of Tirgu Mures through Internal Research Grant $5 / 23.12 .2014$.

\section{Conflict of interest}

None to declare.

\section{References}

1. Brant, William E. Fundamentals of Diagnostic Radiology. [ed.] William E. Brant and Clyde A. Helms. 3rd. s.I. : Lippincott Williams \& Wilkins, 2007:7-10.

2. J. Rydberg, et al. Multisection CT: scanning techniques and clinical applications. RadioGraphics 2000;20:1787-1806.

3. DD Cody. Image processing in CT. RadioGraphics 2002;22:1255-1268.

4. CT. Rachel S. Title, Keith Harper, Erik Nelson, Tom Evans, and Richard Tello. Observer Performance in Assessing Anemia on Thoracic. American Journal of Roentgenology, 2005;185:1240-1244 .

5. Measurements, International Commission on Radiation Units and. Tissue Substitutes in Radiation Dosimetry and Measurement. ICRU. Bethesda: s.n., 1989. 44.

6. EM Kamel, et al. Radiological profile of anemia on unenhanced MDCT of the thorax. Eur Radiol. 2008;18:1863-8.

7. New PF and Aronow S. Attenuation measurements of whole blood and blood fractions in computed tomography. Radiology 1976;121:635-40.

8. AJ Collins, S Gillespie and BE Kelly. Can computed tomography identify patients with anaemia? Ulster Med J. 2007;116-18.

9. DF Black, et al.Cerebral venous sinus density on noncontrast CT correlates with hematocrit. AJNR Am J Neuroradiol. 2011;32:1354-7.

10. Bruni SG, Patafio FM, Dufton JA, Nolan RL, Islam O. head. The assessment of anemia from attenuation values of cranial venous drainage on unenhanced computed tomography of the. 1. Can Assoc Radiol J. 2013;64:46-50. 